\title{
AN IMPROVED DISCRETISATION METHOD FOR BOUNDED CONVECTIVE SCHEMES ON UNSTRUCTURED CO-LOCATED GRIDS
}

\author{
V. PRŽULJ \\ Ricardo Software, Ricardo UK Ltd, Shoreham-by-Sea, West Sussex, BN43 5FG, UK \\ e-mail: vlado.przulj@ ricardo.com, web page: www.ricardo.com/en-GB/What-we-do/Software
}

Keywords: CFD, Convection, Bounded Scheme, CBC/TVD Criteria, Finite-Volume, Colocated Grid, Unstructured Grid.

\begin{abstract}
.
In comparison to structured grids, a more refined discretisation of convective transport on unstructured grids is required to account for their complex topologies. In particular, and with reference to the given cell-face, the far upwind node is not readily available on unstructured grids. Also, the solution accuracy can be affected by numerical diffusion caused by skewness of the grid. In this paper, a novel method is introduced for the far upwind node reconstruction in the framework of the cell-centred finite-volume discretisation. The main idea is to form an upwind-biased stencil with a local axis that connects the central cell node and the geometric cell-face centre. Note that central and downwind cells share the given cell-face. Instead of the actual downwind node, its projection onto the local axis is used. In the previous methods, the local axis has been defined along the line connecting the central and downwind nodes. These methods can not mitigate the grid skewness error whereas by its definition the new method bypasses the issue of grid skewness. The present method is applied to several test cases, covering transport of a scalar step-profile, laminar vortex shedding from a circular cylinder and turbulent flows in two engine intake ports. Depending on the test-case, the high resolution convective schemes such as MINMOD, the modified SMART and bounded central differencing scheme are employed. The latter is selected for large eddy simulations of one of engine ports. The preceding schemes are formulated for non-uniform grids, and they obey the well known convective boundedness criteria. The used grid types also varied, including triangular, polyhedral and Cartesian cut-cell shapes. The convergence properties and accuracy of the new method are scrutinised against the previous one. For all cases, the bounded numerical solution is ensured, and the improved convergence rate of the new method is noted. The accuracy of results predicted by both methods is established through comparisons with either analytical or experimental and numerical results from literature.
\end{abstract}




\section{INTRODUCTION}

Discretisation of fluid transport equations should ensure a convergent, accurate and physically bounded numerical solution. Closely related to the solution accuracy is the perplexing problem of boundedness. The difficulty is that proper bounds exist for any numerical problem but these bounds are not always easily perceived. The boundedness problem particularly affects the discretisation of convective transport on either structured or unstructured grids.

Calculation of cell-face variable values using linear interpolation between adjacent cell values, which is known as central differencing scheme (CDS), illustrates well the boundedness problem. If the numerical grid is not sufficiently refined this scheme can not suppress numerical oscillations accompanied by non-physical overshoots or undershoots, especially in regions with steep gradients of transported variables. This behaviour is predicted by Godunov's theorem [1] which states that the second and higher order linear schemes can not be monotonicity preserving. Only the first order accurate upwind differencing scheme (UDS) guarantees the solution boundedness whereby the cell-face value is taken to be that which prevails at the upwind cell. In order to maintain the bounded solution of the second and higher order convection schemes, these schemes should be designed as non-linear schemes, with provision of an appropriate amount of numerical diffusion.

The way in which the numerical diffusion is introduced, and its amount controlled, determines the accuracy, convergence properties and computational efficiency of the convective (numerical) scheme. A popular approach is to control the numerical diffusion by boundedness criteria. Two major criteria, total variation diminishing (TVD) $[2,3]$ and the convection boundedness criterion (CBC) [4], offer great flexibility in the construction and implementation of high resolution bounded schemes. These two criteria were formulated in terms of flux limiters and normalised variables, respectively. The review and comparison of TVD and CBC criteria, together with analysis of most higher-order bounded schemes can be found in [5].

In the framework of the cell-centred, finite-volume discretisation, each cell-face value is calculated with the help of a dimensionally split upwind-biased stencil, i.e. one-dimensional bounded scheme is applied along appropriately defined cell-face direction. On the structured, in general non-uniform and non-orthogonal grids, the upwind-biased stencil is defined along the local coordinates (grid lines) as shown in Figure 2 (left) for the east cell-face. The stencil involves the upwind $U$ and downwind $D$ cells (nodes) with respect to the cell $C$ which is itself upwind of the face $j$. The implementation of bounded schemes on multi-dimensional structured grids is relatively straightforward. Though it is very important to formulate these schemes in terms of non-uniform grid spacing where either normalised local coordinates [6, 7] or equivalent downwind and upwind geometric factors [8, p.48] can be used.

Considering unstructured grids, the implementation of the TVD/CBC schemes is neither straightforward nor unique task as the upwind node is not readily available. A sensible idea is to reconstruct the upwind node and its value. This can be done implicitly by projecting the central cell variable gradient onto the line connecting the central and downwind nodes as devised by $[9,10,11]$. Instead of the central cell gradient, the projection of the cell-face gradient in conjunction with the modified definition of the CBC normalised variable was employed in [12]. Both approaches are equivalent as they deliver the same definition of the normalised variables, and the actual position of the upwind node is not required. It was shown by $[10,11]$ that reconstructed upwind values should be bounded by maximum and minimum values of central node's neighbours. The effectiveness of upwind node reconstruction using formulation for the linear CBC/TVD schemes consistent with non-uniform grids was demonstrated in study 
[11]. Alternative methods rely on the explicit definition of the upwind node, and require a geometric search to find an upwind cell closest to the defined upwind node. Then the upwind node value is the value at the upwind cell corrected with the help of the upwind cell gradient. In [13], the upwind node was defined in the same way as in the gradient projection method. Other authors, [14, 15], positioned the upwind node along the line normal to the cell-face. This line passes through the cell-face centre as well as through the nodes $C^{\prime}$ and $D^{\prime}$ which are projections of the $C$ and $D$ nodes onto the face normal, respectively. The recent multi-slope MUSCL method [16] specifies the upwind node along the line connecting the central node and cell-face centre. In essence, the upwind value is obtained by linear interpolation from the values available at the closest cell centres.

The majority of previous upwind node reconstruction methods calculated the bounded cellface value at the cell-face interpolation point $j^{\prime}$. This intersection point, defined by vector connecting the central and downwind nodes, did not always coincide with the face geometric centre $j$. Consequently, these methods, apart from [16], could not fully alleviate the grid skewness interpolation error. In the methods employing the line normal to the cell-face $[14,15]$, the interpolation point was the cell-face centre. However, the variable values and gradients at projected central and downwind nodes were taken to be those at the corresponding actual nodes. The skewness corrected TVD flux limiter, proposed recently by [17], was devised to correct the cell-face value implicitly. The limiter itself must be carefully bounded to satisfy the TVD constraints.

In this paper, a novel method, illustrated in Figure 2 (right), is proposed to mitigate the grid skewness error explicitly. For this, the upwind node $U$ is reconstructed along the cellface direction defined by line connecting the central node $C$ and the face centre $j$. Instead of the actual downwind node $D$, its projection onto the cell-face direction, $D^{\prime}$, is used. After an overview of the finite volume discretisation, bounded convective schemes are introduced with the help of normalised variable approach. Next, a new generalised implementation of convective schemes on unstructured grids is described. Then the results from four test cases are used to assess the performance of the proposed method.

\section{OVERVIEW OF FINITE VOLUME DISCRETISATION}

The discretisation of the governing equations is carried out by using the finite volume method to discretise spatial dimensions and finite differencing to discretise time. The solution domain is filled with an unstructured grid defined by edges of arbitrary polyhedra referred to as control volumes or cells. All flow variables are stored at geometric centres of the cells. For a nonmoving control volume $V$, depicted in Figure 1, integration of fluid transport equations using second-order single-point quadrature operators leads to control volume balances of unsteady, convective, diffusive and source terms, $[18,19]$ :

$$
\begin{gathered}
\frac{d}{d t}(\rho V \phi)_{P}+\sum_{j=1}^{n_{f}}\left(C_{j}-D_{j}-\left(s_{\phi k}^{A} A_{k}\right)_{j}\right)=\left(s_{\phi}^{V}\right)_{P} V_{P}, \\
\text { with } C_{j}=\dot{m}_{j} \phi_{j}, D_{j}=\left(\Gamma_{\phi} \nabla \phi \cdot \vec{A}\right)_{j}
\end{gathered}
$$

In the preceding equation, $\phi$ is a flow variable and $\Gamma_{\phi}$ represents its diffusion coefficient; time and density are denoted as $t$ and $\rho$, respectively. For a cell-face $j$, convective and diffusion fluxes are $C_{j}$ and $D_{j}$, respectively. The source terms $s_{\phi}^{V}$ and $s_{\phi}^{A}$ are related to the cell volume and cell face, respectively; $n_{f}$ is the number of cell-faces enclosing the cell. Mass fluxes $\dot{m}_{j}$ are 


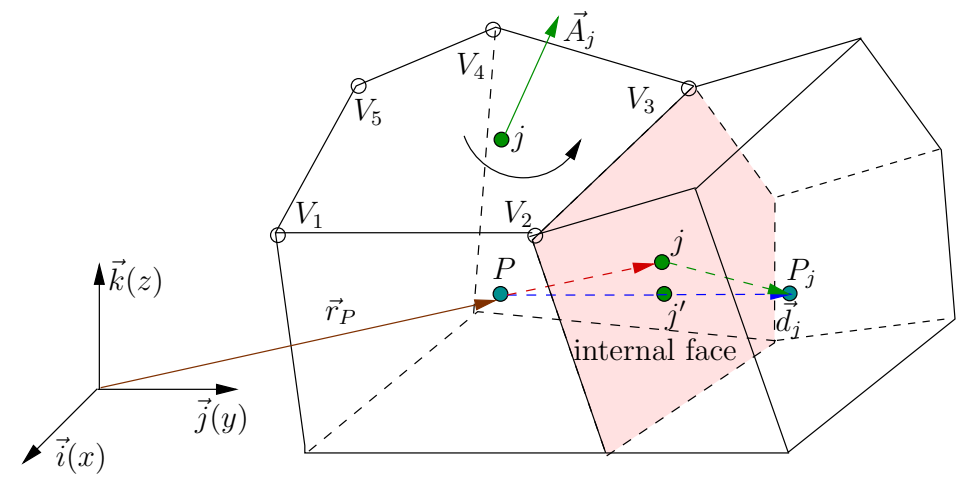

Figure 1: A polyhedral control volume (cell) $P$ as enclosed by its cell-faces $j$. A cell-face $j$, with the outward normal vector $\vec{A}_{j}$, is defined by a list of vertices $V_{k}$.

defined as:

$$
\dot{m}_{j}=\rho_{j} \vec{U}_{j}^{*} \cdot \vec{A}_{j}
$$

where the Rhie-Chow interpolation practice $[20,21]$ is used for the mass conserving face velocity $\vec{U}_{j}^{*}$. The mass fluxes must satisfy the integral mass balance over each cell:

$$
\frac{d}{d t}(\rho V)_{P}+\sum_{j=1}^{n_{f}} \dot{m}_{j}=0
$$

For time-dependent phenomena, the semi-discrete equation (1) can be written in a form that resembles the first order ordinary differential equation. Time 'marching' procedure is then used to advance the solution in time over a number of time steps with the time-step size $\Delta t$. The unsteady term is integrated over each time interval by either first order Euler or second order three time level (TTL) differencing scheme, see [21]. Both schemes are implicit and unconditionally stable.

The evaluation of cell-face values and gradients at both cell-centres and cell-faces is the essential ingredient of the discretisation procedure. Commonly used linear interpolation between the values at adjacent cells $P$ and $P_{j}$ (which amounts to the central differencing scheme, CDS) gives

$$
\begin{gathered}
\phi_{j}=f_{j} \phi_{P}+\left(1-f_{j}\right) \phi_{P_{j}} \\
f_{j}=f_{j, P}=\frac{\left|\vec{r}_{P_{j}}-\vec{r}_{j}\right|}{\left|\vec{r}_{P_{j}}-\vec{r}_{j}\right|+\left|\vec{r}_{j}-\vec{r}_{P}\right|}
\end{gathered}
$$

where $f_{j}$ is the cell-face interpolation factor. In addition to CDS, higher-order upwind schemes such as linear upwind (LUDS) [22] and quadratic upwind (QUICK) [23] (generalised by [24] as $\kappa$-schemes) are popular. As pure $\kappa$-schemes are not always appropriate for the convective transport, a simple and efficient approach is to blend them with a certain amount of the first order upwind (UDS) scheme as advocated by $[21,25]$. In the case of CDS, the blending can be arranged as:

$$
\begin{aligned}
& \phi_{j}=\phi_{j}^{U D S}+\gamma_{\phi} f_{j}^{*} \frac{\dot{m}_{j}}{\left|\dot{m}_{j}\right|}\left(\phi_{P_{j}}-\phi_{P}\right) \\
& \phi_{j}^{U D S}=\left\{\begin{array}{ccc}
\phi_{P_{j}} & \text { if } \quad \dot{m}_{j}<0 \\
\phi_{P} & \text { if } \quad \dot{m}_{j} \geq 0
\end{array}, f_{j}^{*}=\left\{\begin{array}{ccc}
f_{j} & \text { if } \quad \dot{m}_{j}<0 \\
1-f_{j} & \text { if } & \dot{m}_{j} \geq 0
\end{array}\right.\right.
\end{aligned}
$$


where $f_{j}^{*}$ is now the flow orientated interpolation factor. The blending factor $\gamma_{\phi}$ depends on the grid resolution, and for sufficiently fine grids the values close to one can be used. The alternative to the blending approach is to adopt high resolution bounded schemes whose improved discretisation on the unstructured grids is the subject of this work.

For the cell gradients, a linear least-square procedure [26] is used in this study. Considering the cell-face gradient, the Gauss' formula $\nabla \phi \approx \oint \phi d \vec{A} / V$ can be applied to the control volume constructed around the face $j$. The derivation outlined in [19] leads to the following expression:

$$
\nabla \phi_{j} \approx \overline{\nabla \phi}_{j}+\frac{\vec{A}_{j}}{\vec{A}_{j} \cdot \vec{d}_{j}}\left[\left(\phi_{P_{j}}-\phi_{P}\right)-\overline{\nabla \phi}_{j} \cdot \vec{d}_{j}\right], \overline{\nabla \phi_{j}}=f_{j} \nabla \phi_{P}+\left(1-f_{j}\right) \nabla \phi_{P_{j}}
$$

Further details regarding the discretisation of convective and diffusion fluxes can be found in $[19,27]$.

The outcome of the discretisation of considered transport equation for $\phi$ is a linearised algebraic equation for each cell. The corresponding system of algebraic equations for each flow variable is under-relaxed implicitly [28]. The system is then arranged in a sparse matrix form $[\mathbf{a}] \phi=\mathbf{b}_{\phi}$, and solved by Bi-CGSTAB linear equation solver $[29,30]$ using incomplete lowerupper (ILU) pre-conditioners.

The non-linearity and coupling of the solved transport equations, including velocitypressure coupling, is handled by generalised SIMPLE-based pressure correction method [31]. This method is suitable for both time marching and steady-state iterative solutions. Normalised residuals, defined as

$$
R_{\phi}=\frac{\sum_{\text {cells }}\left|a_{P} \phi_{P}-b_{\phi}\right|}{\sum_{\text {cells }}\left|a_{P} \phi_{P}\right|}, R_{\text {mass }}=\frac{\sum_{\text {cells }} \mid \text { mass imbalance } \mid}{\sum_{\text {cells }} \mid \text { mass imbalance }\left.\right|_{\text {ref }}}
$$

serve as the convergence criterion for the SIMPLE-based iterative solution procedure. The reference mass imbalance in the preceding equation is the maximum imbalance in the first five iterations [32]. The solution is considered converged when all normalised residuals drop below a certain value, typically below $1 . \times 10^{-5}$.

\section{BOUNDED CONVECTIVE SCHEMES - NORMALISED VARIABLE APPROACH}

High-resolution convective schemes require an upwind-biased stencil as defined along local coordinate $\xi$ in Figure 2 (left) for the structured grid. In this figure, the far upwind $U$, central $C$ and downwind $D$ nodes are defined with respect to the cell-face $j$ having positive mass flow rate $\dot{m}_{j}$. Obviously, labelling of the nodes depends on the mass flux direction. As shown in [11], the mathematical enforcement of monotonicity along local axis $\xi$ and interpolative boundedness (face value bounded by values at adjacent cells) is an easy way to derive both CBC [4] and TVD [3] boundedness criteria. These criteria, and bounded schemes based on them, can be formulated in terms of normalised variable approach introduced by $[4,33]$.

\subsection{Normalised variable approach}

In this approach, the convected variable $\phi$ is normalised as

$$
\widetilde{\phi}=\frac{\phi-\phi_{U}}{\phi_{D}-\phi_{U}}
$$

giving $\widetilde{\phi}_{U}=0$ and $\widetilde{\phi}_{D}=1$. The associated normalised variable diagrams (NVD), showing TVD (left) and CBC (right) regions, are presented in Figure 3. It follows that cell-face values 


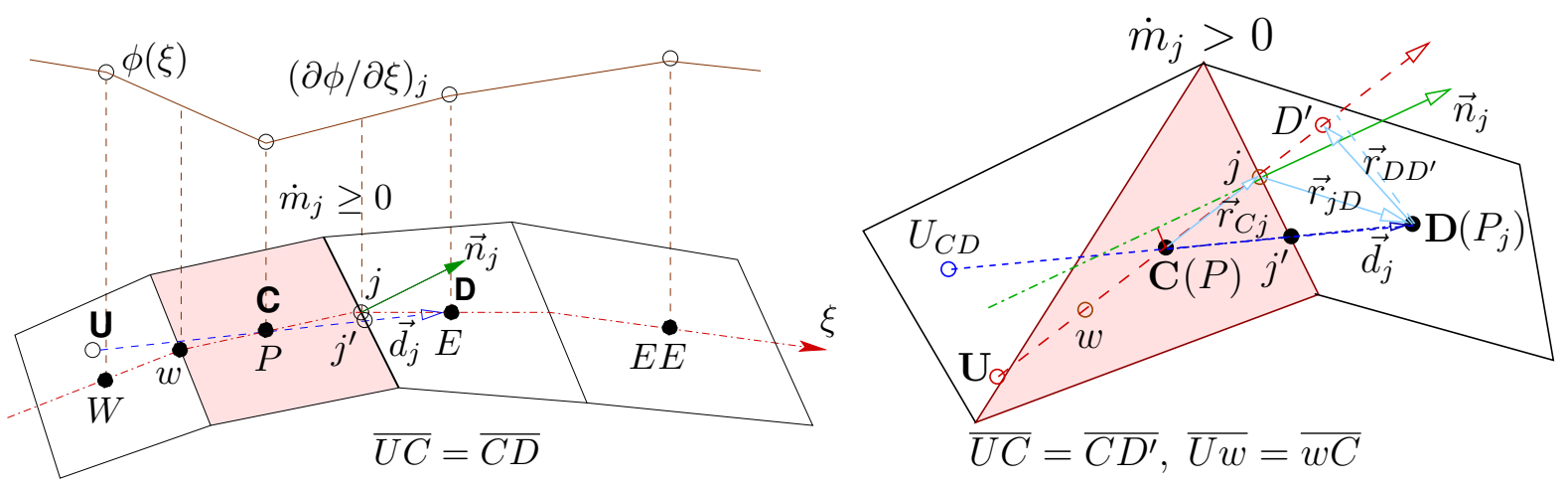

Figure 2: Upwind-biased stencil for structured (left) and unstructured (right) grids.

$\widetilde{\phi}_{j}$ should lie within the shaded areas (monotonic range $0<\widetilde{\phi}_{C}<1$ ), and on the line $\widetilde{\phi}_{j}=\widetilde{\phi}_{C}$ outside the monotonic range. Further, the convective schemes with linear characteristics such as $\kappa$-schemes (CDS, LUDS and QUICK) may violate boundedness criteria. Therefore bounded schemes have to be non-linear or piecewise linear schemes.

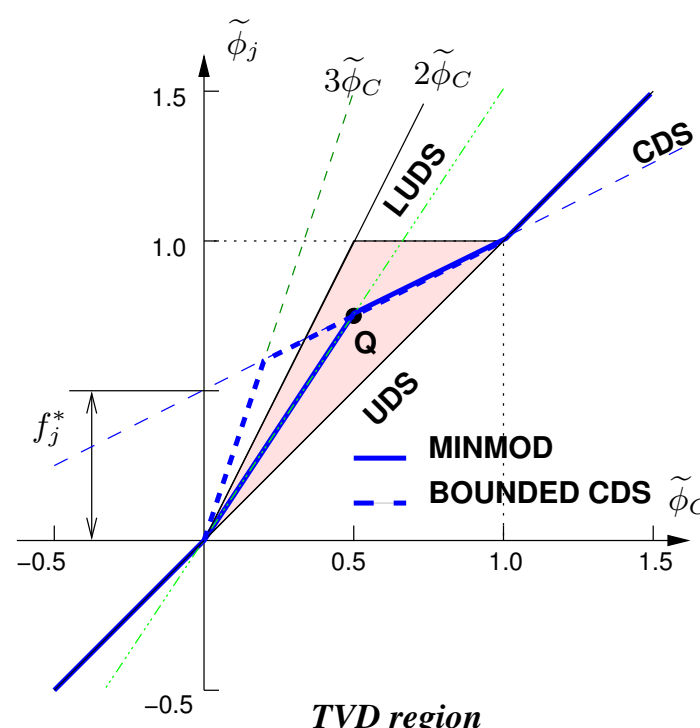

TVD region

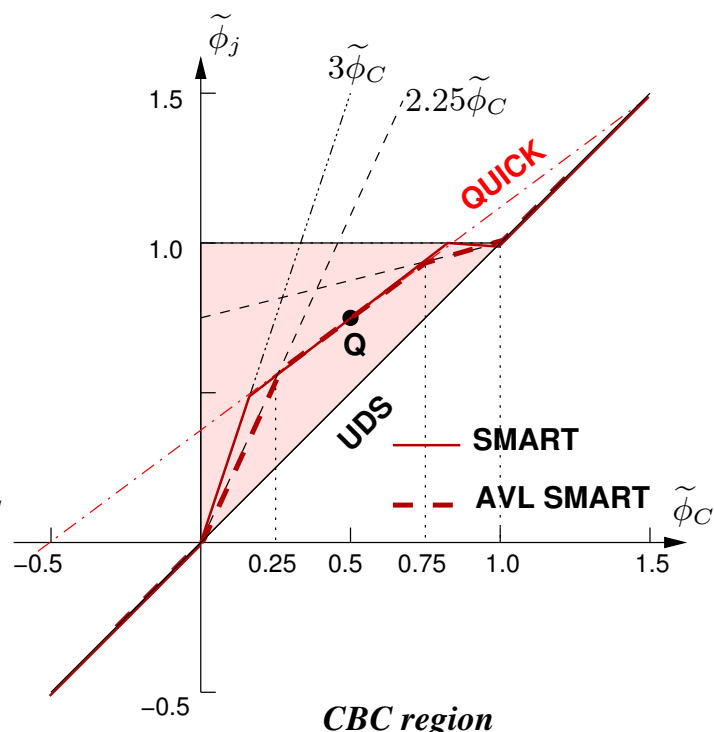

CBC region

Figure 3: NVD approach. TVD (left) and CBC (right) regions and characteristics of well known convective schemes.

It is instructive to generalise linear schemes such as CDS, LUDS and QUICK on the structured non-uniform grids, Figure 2 (left), with the help of flow orientated interpolation factors as done in $[8, \mathrm{p} .48]$ :

$$
\begin{gathered}
\phi_{j}=\phi_{j}^{U D S}+\varphi_{j} \frac{\dot{m}_{j}}{\left|\dot{m}_{j}\right|}\left(\phi_{P_{j}}-\phi_{P}\right)=\phi_{C}+\varphi_{j}\left(\phi_{D}-\phi_{C}\right) \\
\varphi_{j}=\left(g_{D}-\alpha_{j} \frac{f_{j}^{*}}{f_{w}^{*}}\right)+\left(g_{U}+\alpha_{j}\right) \frac{\phi_{C}-\phi_{U}}{\phi_{D}-\phi_{C}} \\
g_{D}=\frac{f_{j}^{*^{2}}\left(1+f_{w}^{*}\right)}{f_{j}^{*}+f_{w}^{*}}, g_{U}=\frac{f_{w}^{*^{2}}\left(1-f_{j}^{*}\right)}{f_{j}^{*}+f_{w}^{*}}, f_{w}^{*}=\left\{\begin{array}{ccc}
f_{j, E} & \text { if } \quad \dot{m}_{j}<0 \\
1-f_{j, W} & \text { if } \quad \dot{m}_{j} \geq 0
\end{array}\right.
\end{gathered}
$$

where the parameter $\alpha_{j}$ defines a family of the higher-order schemes. For example, the QUICK scheme is recovered for $\alpha_{j}=0$. The quantity $\varphi_{j}$ plays a similar role as the flux limiter [3]. In 
addition to geometric interpolation factors, the flux limiter is a function of the gradient ratio $r_{j}$ :

$$
r_{j}=\frac{\phi_{C}-\phi_{U}}{\phi_{D}-\phi_{C}}=\frac{\widetilde{\phi}_{C}}{1-\widetilde{\phi}_{C}} \approx \frac{(\partial \phi / \partial \xi)_{w}}{(\partial \phi / \partial \xi)_{j}}
$$

It is evident from Equation (12) that all NVD schemes $\widetilde{\phi}_{j}=f\left(\widetilde{\phi}_{C}\right)$ can be formulated in terms of flux limiters:

$$
\widetilde{\phi}_{j}=\widetilde{\phi}_{C}+\varphi_{j}\left(1-\widetilde{\phi}_{C}\right)
$$

Note that Equation (11) for the cell-face value offers a straightforward implementation of NVD schemes.

\subsection{Choice of bounded schemes}

Characteristics of bounded schemes used in this study are shown in Figure 3. In comparison to the original SMART scheme [4], the AVL SMART [11] offers a good compromise between accuracy and convergence properties. Both schemes coincide with the third-order accurate QUICK scheme over a large part of the monotonic region. The TVD MINMOD scheme (Roe's limiter) is a combination of the second-order accurate LUDS and CDS schemes. It is more diffusive than the AVL SMART/SMART scheme, and therefore has better convergence properties.

With exception of implicit large eddy simulations (LES), the CDS scheme is an ideal choice for LES on the adequately resolved numerical grids, [34]. Unfortunately, achieving the good grid resolution is not always practical. To compensate for inadequate grid resolution, and maintain the numerical stability and boundedness, the bounded CDS (BCDS) scheme has been devised. As shown in Figure 3 (left), this composite scheme has a central differencing behaviour for $0.2 \leq \widetilde{\phi}_{C} \leq 1$.

The upwind node for bounded schemes on unstructured grids is usually placed at the same distance from the central node as the downwind node. For this situation, the flow orientated interpolation factor at the cell-face $w$ in Equation (13) is the same as at cell-face $j, f_{w}^{*}=f_{j}^{*}$. The corresponding flux limiters are then defined as:

MINMOD: $\quad \varphi_{j}=f_{j}^{*} \max \left[0, \min \left(r_{j}, 1\right)\right]$

Bounded CDS: $\quad \varphi_{j}=f_{j}^{*} \max \left[0, \min \left(4 r_{j}, 1\right)\right]$

AVL SMART:

$$
\begin{aligned}
& \varphi_{j}=f_{j}^{*} \max \left\{0, \min \left[\left(1+\beta_{1}\right) r_{j}, \frac{1}{2}\left(\beta_{1}+\beta_{2} r_{j}\right), 1+\beta_{2}\right]\right\} \\
& \beta_{1}=1+f_{j}^{*}, \beta_{2}=1-f_{j}^{*}
\end{aligned}
$$

\section{GENERALISED IMPLEMENTATION OF BOUNDED SCHEMES}

As outlined in the Introduction, original implementations of unstructured grid schemes employed an upwind-biased stencil whose axis was defined by a line connecting central $C$ and downwind $D$ nodes, Figure 2 (left). The acronym UCD-method will be used to refer to this type of upwind node reconstruction. In this case, the centred difference $\left(\phi_{D}-\phi_{U}\right)$ can be calculated from the gradient projection $\nabla \phi_{C} \cdot 2 \vec{d}_{C D}$, which gives the upwind node value $\phi_{U}^{*}$ as:

$$
\phi_{U}^{*}=\phi_{D}-\nabla \phi_{C} \cdot 2 \vec{d}_{C D}
$$


The preceding gradient projection implies a uniform stencil, that is virtual upwind node $U$ is at the same distance from $C$ as $C$ is from $D$. Also, gradient changes between upwind and downwind nodes are neglected.

The grids shown in Figure 2 are not only non-orthogonal but also skewed as the distance vector, connecting the central and downwind nodes, intersects the cell-face at an interpolation point $j^{\prime}$ which does not coincide with the face geometric centre $j$. As a consequence, the UCDmethod provides the cell-face value $\phi_{j}$ at the interpolation point $j^{\prime}$. Grid skewness is a common feature of unstructured grids. The associated error, analysed by [35], appears as a diffusion-like error. It can be easily corrected in an explicit manner [35, 36] as follows:

$$
\phi_{j}=\phi_{j \prime}+\nabla \phi_{j} \cdot\left(\vec{r}_{j}-\vec{r}_{j \prime}\right)
$$

The above correction, however, may produce an unbounded value at the cell-face centre.

A new method, illustrated in Figure 2 (right), adopts the line connecting the central cell $C$ and cell-face centre $j$ as a local coordinate for the upwind-biased stencil. This method, referred to as $U C F-$ method, bypasses the grid skewness issue. However, a projection $D^{\prime}$ of the downwind node $D$ onto the stencil axis as well as a variable value for this projected node are required. An orthogonal projection of $D$ appears to be a good choice as it minimises an interpolation error for the projected node value $\phi_{D^{\prime}}^{*}$ :

$$
\phi_{D^{\prime}}^{*}=\phi_{D}+\nabla \phi_{D} \cdot\left(\vec{r}_{D^{\prime}}-\vec{r}_{D}\right)
$$

Similar to the UCD-method, the upwind node $U$ and projected node $D^{\prime}$ are equidistant to node $C$. The interpolation factor for the cell-face $j$ is calculated from:

$$
f_{C j}^{*}=\frac{\overline{C j}}{\overline{C D^{\prime}}}=\frac{\overline{C j}}{\overline{C j}+\overline{j D^{\prime}}}=\frac{\vec{r}_{C j} \cdot \vec{r}_{C j}}{\vec{r}_{C j} \cdot \vec{r}_{C j}+\left|\vec{r}_{j D} \cdot \vec{r}_{C j}\right|}
$$

The position vector of the projected node is then:

$$
\vec{r}_{D^{\prime}}=\vec{r}_{C}+\frac{\vec{r}_{C j}}{f_{C j}^{*}}
$$

Clearly, the position of projected node can be controlled by interpolation factor value. One can be tempted to define this value equal to $1 / 2$, i.e to design the upwind stencil as uniform. Numerical tests, however, revealed that biasing of interpolation factors towards $1 / 2$ as in:

$$
f_{j}^{*}=\frac{1}{2}\left(f_{C j}^{*}+\frac{1}{2}\right)
$$

improves the convergence rate without the negative impact on the accuracy. Thus, the preceding definition of the flow orientated cell-face interpolation factor is used for flux limiters in Equations (16), (17) and (18), whereas the projected node $D^{\prime}$ is placed according to Equation (23) using $f_{j}^{*}$ instead of $f_{C j}^{*}$.

Another novelty of the current method is a calculation of the upwind node value using gradient projection at an imaginary cell-face $w$ :

$$
\phi_{C}-\phi_{U}^{*}=\frac{1}{f_{j}^{*}} \nabla \phi_{w} \cdot \vec{r}_{C j} \Rightarrow \phi_{U}^{*}=\phi_{C}-\frac{1}{f_{j}^{*}} \nabla \phi_{w} \cdot \vec{r}_{C j}
$$


The required gradient at $w$ is reconstructed from the assumed linear distribution of gradients across the central cell (involving points $w, C$ and $j$ ):

$$
\nabla \phi_{w}=\frac{1}{2 f_{j}^{*}}\left[\left(1+2 f_{j}^{*}\right) \nabla \phi_{C}-\nabla \phi_{j}\right]
$$

where the variable gradient $\nabla \phi_{j}$ at the cell-face $j$ is given by Equation (8).

Both upwind node and projected downwind node values are bounded by values at neighbouring cells that surround the upwind and downwind node, respectively. This is done by checking $\phi_{U}^{*}$ and $\phi_{D^{\prime}}^{*}$ against the minimum and maximum values of $\phi$ over the cells that share faces of $C$ and $D$, respectively:

$$
\phi_{U}=\max \left[\phi_{\min }^{C n g b}, \min \left(\phi_{U}^{*}, \phi_{\max }^{C n g b}\right)\right], \phi_{D^{\prime}}=\max \left[\phi_{\min }^{D n g b}, \min \left(\phi_{D^{\prime}}^{*}, \phi_{\max }^{D n g b}\right)\right]
$$

The convected variable $\phi$ is now normalised by replacing the downwind value $\phi_{D}$ in Equation (10) with the projected node value $\phi_{D^{\prime}}$.

\section{RESULTS AND DISCUSSION}

The test cases used in this study include pure convection of scalar discontinuities, laminar vortex shedding from a circular cylinder at $R e=105$, turbulent flow through an engine port and LES of the JSAE engine port. Unstructured grids made of triangular, polyhedral and Cartesian cut cells are employed. The convergence properties and accuracy of the new method are scrutinised for all test cases.

\subsection{Convection of step profile}

A steady-state convective transport of a passive scalar, $\nabla \cdot \rho \vec{U} \phi=0$, is frequently used to test and validate convective schemes. Here, a step profile is convected over a square domain by the given velocity field $\vec{U}=(\sqrt{2} / 2, \sqrt{2} / 2)$. Figure 4 (left) shows the geometry and boundary conditions for this case. A mildly skewed triangular grid, having 25 cells per side of a square, is used, Figure 4 (right)).

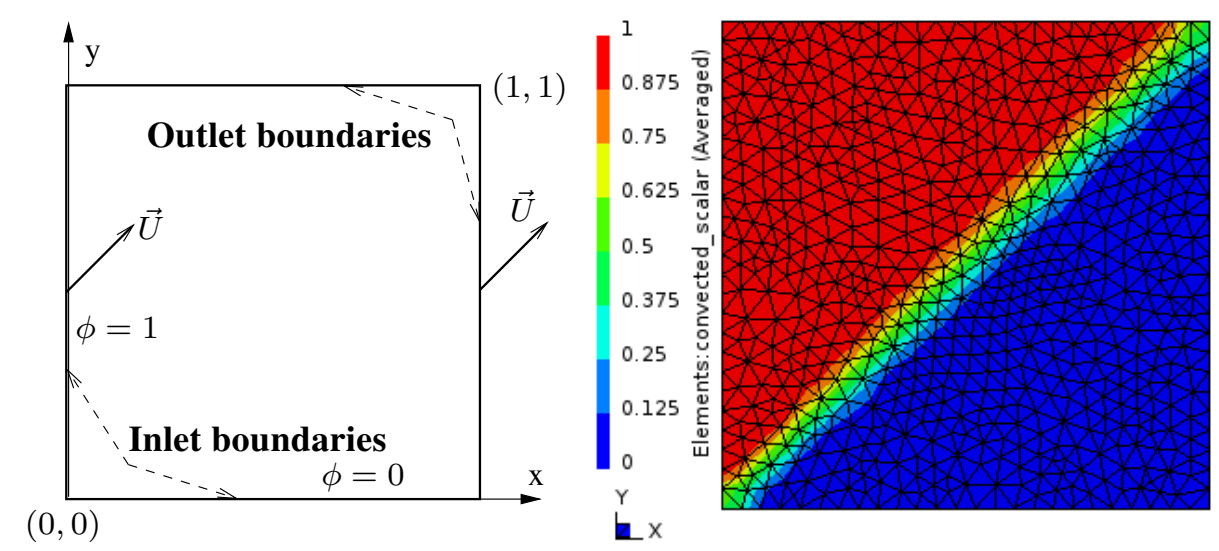

Figure 4: Step profile. Geometry with boundary conditions (left) and scalar distribution as computed by AVL SMART scheme (right).

The results were obtained by using double precision variables, and the convergence criterion based on the normalised residuals was $1 \cdot 10^{-14}$. As Figure 5 indicates, the new UCF upwind node reconstruction method has a better convergence rate than the previous UCD method. In 


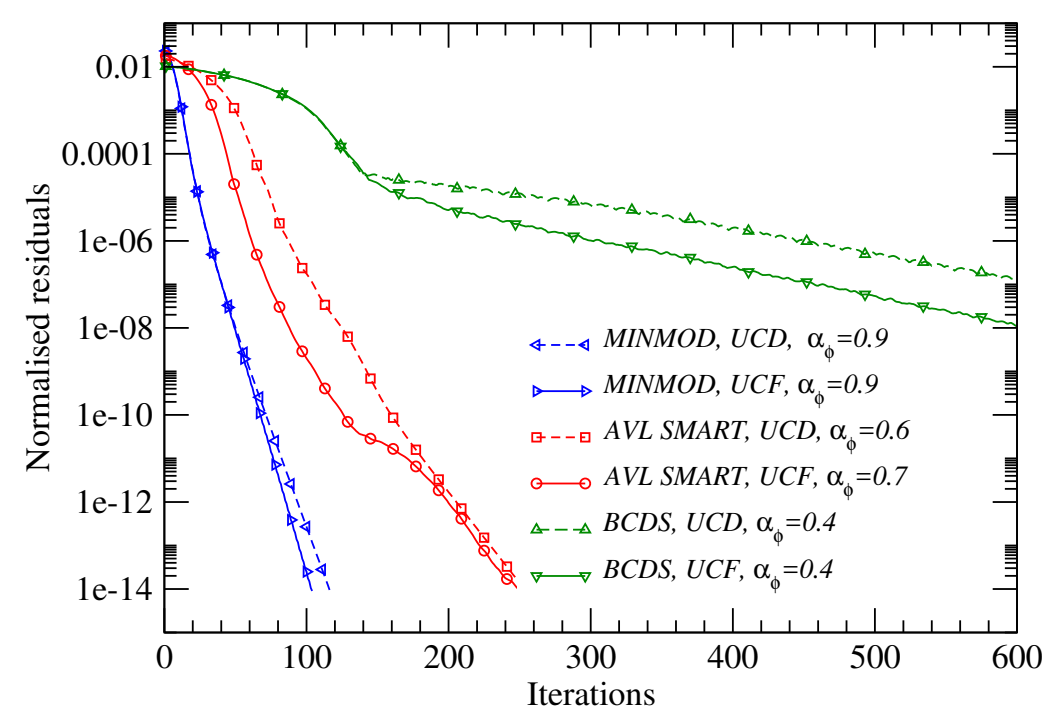

Figure 5: Step profile. Effect of upwind-node reconstruction methods on the convergence rate.

general, more accurate schemes require smaller under-relaxation factors $\alpha_{\phi}$ and more steadystate iterations to achieve the convergence. Though the bounded CDS has converged below the specified convergence criterion, its convergence properties in this case are not great.

The comparison of all schemes in terms of their ability to resolve the step profile is shown in Figure 6. Regardless of the upwind node reconstruction method, more accurate schemes

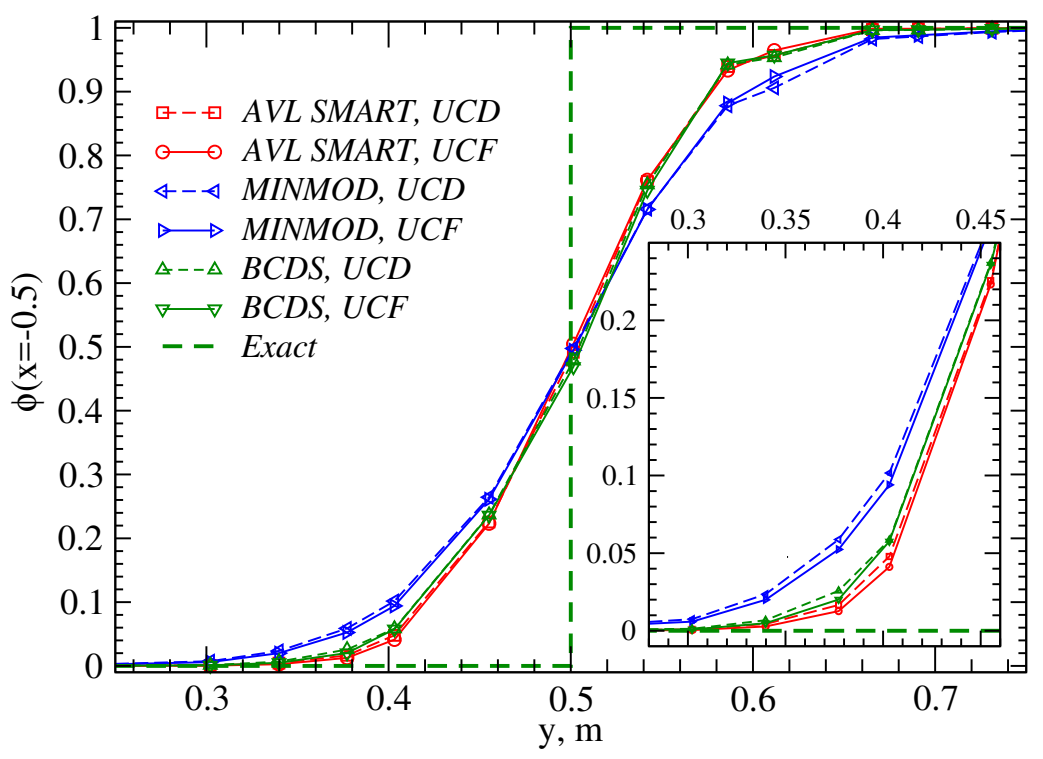

Figure 6: Step profile. Effect of upwind-node reconstruction methods on the scalar profile resolution at $x=0.5$.

deliver better resolutions. The bounded CDS capability to resolve the step profile is quite close to AVL SMART, and significantly better than of MINMOD. Clearly, the proposed UCF method performs better than the UCD method for all tested schemes.

\subsection{Laminar vortex shedding from a circular cylinder}

The incompressible, unsteady flow around a two-dimensional circular cylinder is computed at Reynolds number $R e=\rho U_{0} D / \mu=105$, where $D=1 \mathrm{~m}$ is the cylinder diameter and 
$U_{0}$ is the uniform inlet velocity. The flow is characterised by the presence of vortex shedding whose predicted strength is very sensitive to the employed convective scheme, [8]. A part of the solution domain, filled with triangles, is displayed in Figure 7. With the reference to the

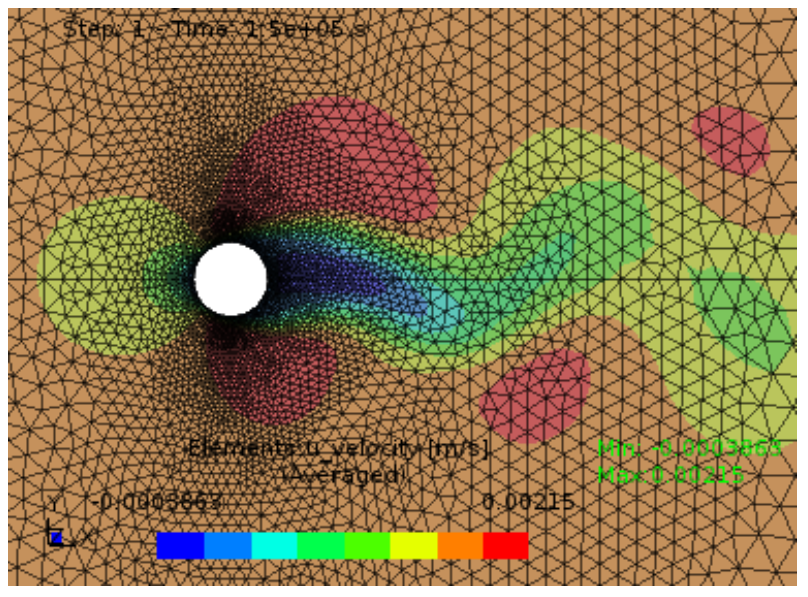

Figure 7: Circular cylinder. Triangular grid and vortex shedding pattern as visualised by distribution of $U$-velocity component at the given time.

cylinder centre, the inlet plane is placed at a distance $12 \mathrm{D}$, the outlet plane at $30 \mathrm{D}$ and the top and bottom symmetry planes at $12 D$. A triangular grid, with 17,504 cells is employed. There are 160 cells along the cylinder wall, with approximate wall distances of $5.75 \times 10^{-3} \mathrm{~m}$.

Computations are performed using the third-order accurate AVL SMART scheme. Both first order Euler and second order TTL time schemes are used with a small time step size of $\Delta t=0.5 \mathrm{~s}$. This gives the non-dimensional time step size $\Delta t^{*}=U_{0} \Delta t / D=8.2 \cdot 10^{-4}$. The convergence criterion is set to $1 . \times 10^{-6}$.

Figure 8 compares the time histories of the lift $\left(C_{L}\right)$ and drag $\left(C_{D}\right)$ coefficients (drag and lift forces normalised by $\left.0.5 \rho U_{0}^{2} D\right)$ as calculated by UCD and UCF upwind node reconstruction methods. Both methods deliver similar histories although the phase shift between two methods
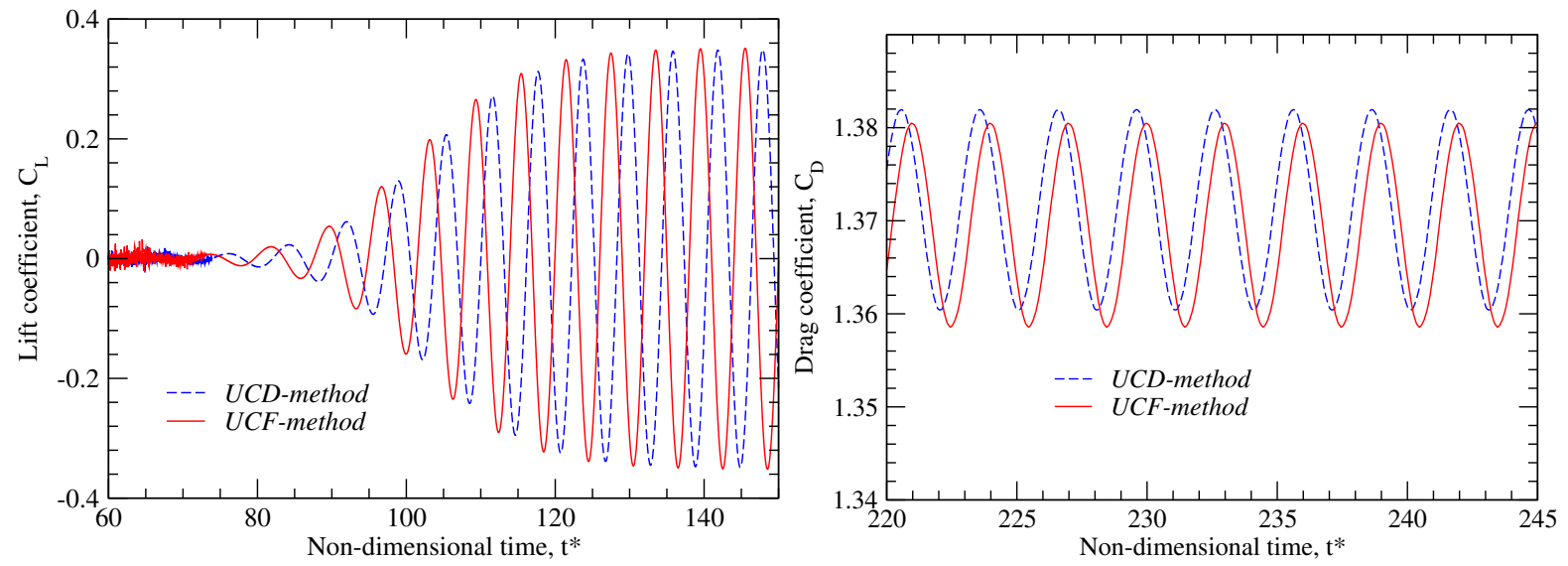

Figure 8: Vortex shedding from a circular cylinder at $R e=105$. Time histories of the lift (left) and drag (right) coefficients as obtained by two upwind node reconstruction methods.

is evident. The integer flow parameters such as Strouhal number $S t$, the time-averaged drag coefficient $\bar{C}_{D}$ and amplitudes of the lift coefficient $\widetilde{C}_{L}$ are compared in Table 1 . For the same time-differencing scheme, the new UCF-method predicts lower values for the mean drag and 
Table 1: Vortex shedding from a circular cylinder at $R e=105$. Integral flow parameters as predicted by two upwind node reconstruction methods.

\begin{tabular}{|l|lll|lll|}
\hline Time scheme & \multicolumn{3}{|c|}{ Euler } & \multicolumn{4}{c|}{ Three time level } \\
Flow parameter & $S t$ & $\bar{C}_{D}$ & $\pm \widetilde{C}_{L}$ & $S t$ & $\bar{C}_{D}$ & $\pm \widetilde{C}_{L}$ \\
\hline \hline UCD-method & 0.1660 & 1.371 & \pm 0.350 & 0.1636 & 1.374 & \pm 0.355 \\
UCF-method & 0.1665 & 1.370 & \pm 0.352 & 0.1638 & 1.369 & \pm 0.355 \\
\hline
\end{tabular}

slightly higher values for the Strouhal number. The differences between the TTL and Euler scheme results are relatively small.

Note that predicted flow parameters are sensitive to the size of the solution domain. Therefore it is important to make a comparison with the results corresponding to the same or similar size of the solution domain. The correlations developed by [37], and based on the polynomial fitting of spectral element method results, are useful in this context as they take into account the solution domain extents. Thus the comparison of present results for a more accurate TTL scheme with the numerical correlations from [37] and the experiment of [38] is given in Table 2 . It can be seen that new UCF-method results are in slightly better agreement with the

Table 2: Vortex shedding from a circular cylinder at $R e=105$. Comparison of predicted integral parameters as obtained by TTL scheme with the results from literature. The values in brackets are relative differences with reference to values from [37, 38].

\begin{tabular}{|l|lcl|}
\hline Authors & $S t$ & $\bar{C}_{D}$ & $\pm \widetilde{C}_{L}$ \\
\hline \hline Present, UCD & $0.1636(-1.9 \%)$ & $1.374(0.4 \%)$ & $0.355(-0.8 \%)$ \\
Present, UCF & $0.1638(-1.7 \%)$ & $1.369(0.0 \%)$ & $0.355(-0.8 \%)$ \\
Experiment, [38] & 0.1667 & - & - \\
Numerical, [37] & - & 1.369 & 0.358 \\
\hline
\end{tabular}

results from literature than the UCD-method results.

\subsection{Steady-state turbulent flow through an engine port}

The turbulent flow trough a baseline intake port with the non-dimensional valve lift $L / D_{i}=$ 0.355 ( $D_{i}$ is the valve inner seat diameter) is computed. The MINMOD convective scheme is employed whereas turbulence is modelled using the time-scale bounded $k-\varepsilon$ model [39] in conjunction with enhanced wall functions [40].

The experimental port assembly is illustrated in Figure 9 (left). The air flow is supplied by an inlet plenum where the stagnation boundary conditions are maintained. The flow leaves a port cylinder via two outlet tubes where impulse torque meters are installed. The torque meters are placed close to outlet tubes ends. Note that $y$-axis starts at the port symmetry plane, and coincides with the outlet tubes axes. From the outlet tubes, the flow is discharged into atmosphere at the constant pressure. The flow experiments are conducted under adiabatic conditions (zero heat fluxes at all walls).

In the present simulations, the solution domain corresponds to the experimental rig flow geometry. However, the outlet tubes are truncated so that outflow pressure boundaries coincide with the starting positions of torque meters in the experimental rig. In this way the flow through the torque meter honeycomb is not modelled as porous media, i.e the presence of meters is ignored. The working fluid is air, and it is considered as an ideal compressible gas for the density predictions. The numerical grid, comprising 2, 638, 706 internal polyhedral cells is used. 

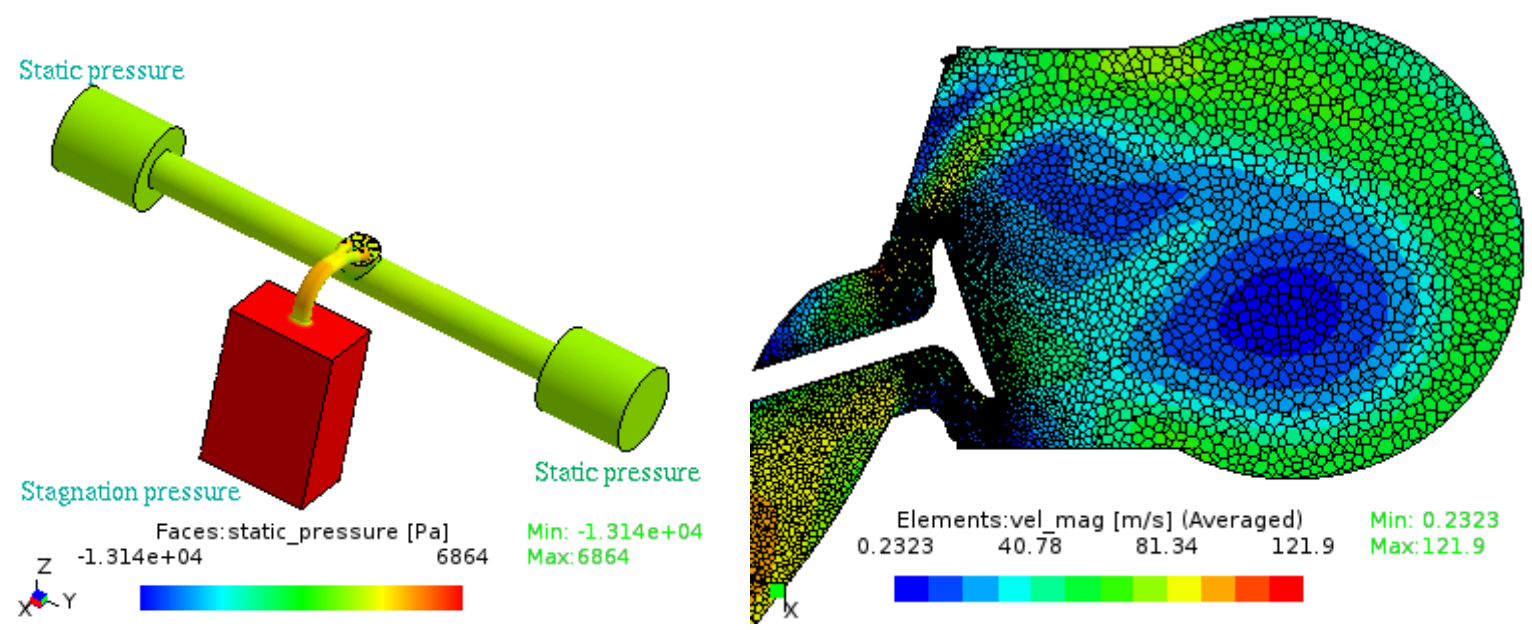

Figure 9: Intake port. Geometry (left) and velocity magnitude at the cross-section $y=20 \mathrm{~mm}$ (right).

Details of this grid around the valve, accompanied with the velocity magnitude distribution, are displayed in Figure 9 (right).

The convergence behaviour of the proposed UCF-method versus the UCD-method can be examined through the history of normalised residuals shown in Figure 10. With the UCF-

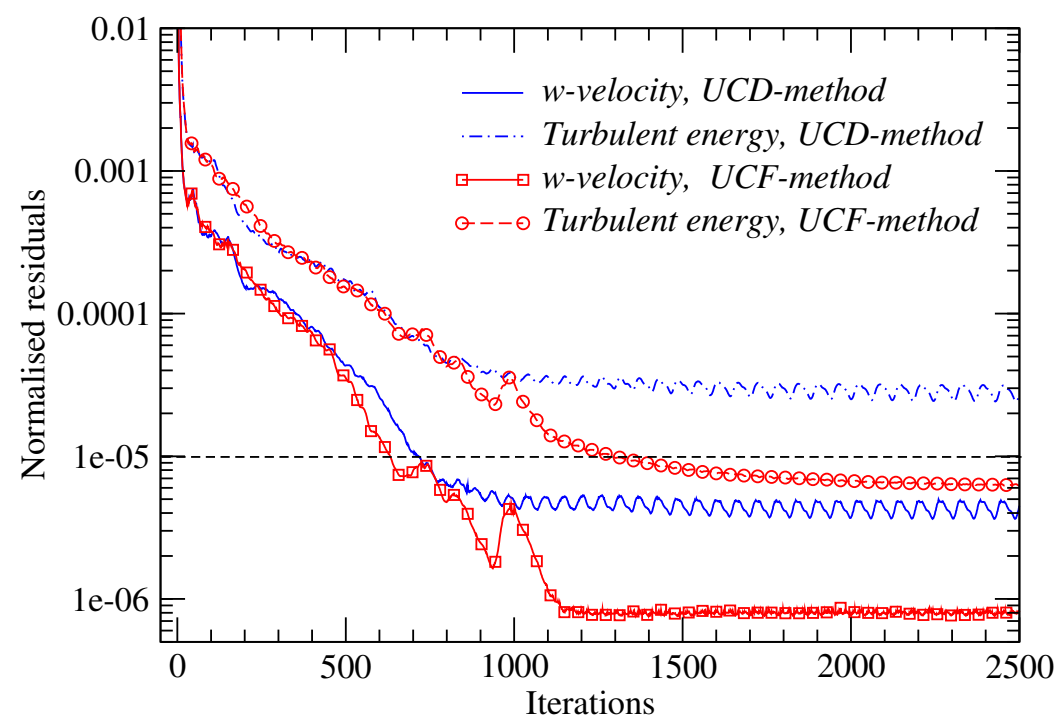

Figure 10: Intake port. Normalised residuals for $w$-velocity and turbulent kinetic energy corresponding to the two upwind node reconstruction methods.

method, the normalised residuals dropped below $1 \times 10^{-5}$ whereas this was not the case for the UCD-method. The calculated values of the inlet mass flow rate $\dot{m}_{i n}$ and the torque $T$ (at the $y$-plane defined by position of a torque meter) are compared with the measured ones in Table 3 . A good agreement between the predicted results with the measured ones is evident. Although the calculated torque value using the UCD-method appears quite close to the measured one, it should not be overlooked that the UCD-method has not fully converged. 
Table 3: Intake port. Comparison of the predicted mass flow rate and torque values with the measured ones.

\begin{tabular}{|l|ccl|}
\hline \hline Quantity & UCD-method & UCF-method & Measured \\
\hline \hline Mass flow rate $\dot{m}_{i n}, \mathrm{~kg} / \mathrm{s}$ & 0.09841 & 0.09875 & 0.10236 \\
Relative difference, \% & -3.86 & -3.53 & - \\
\hline Torque $T \times 10^{3}, \mathrm{Nm}$ & 27.752 & 27.008 & 28.239 \\
Relative difference, $\%$ & -1.72 & -4.35 & - \\
\hline \hline
\end{tabular}

\subsection{Large-eddy simulation of JSAE port}

In this validation case, LES of JSAE engine port is performed using Smagorinsky-Lilly sub-grid scale model and bounded CDS scheme.

The port model was designed by Japanese Society of Automotive Engineers (JSAE) for their benchmark study, see [41]. Its geometry, shown in Figure 11 (left), has a simple $90^{\circ}$ axisymmetric design which provides the non-swirling flow around an engine valve at a lift of $7 \mathrm{~mm}$ At the port inlet, the mass flow rate of $0.0254 \mathrm{~kg} / \mathrm{s}$ was prescribed, while the static pressure
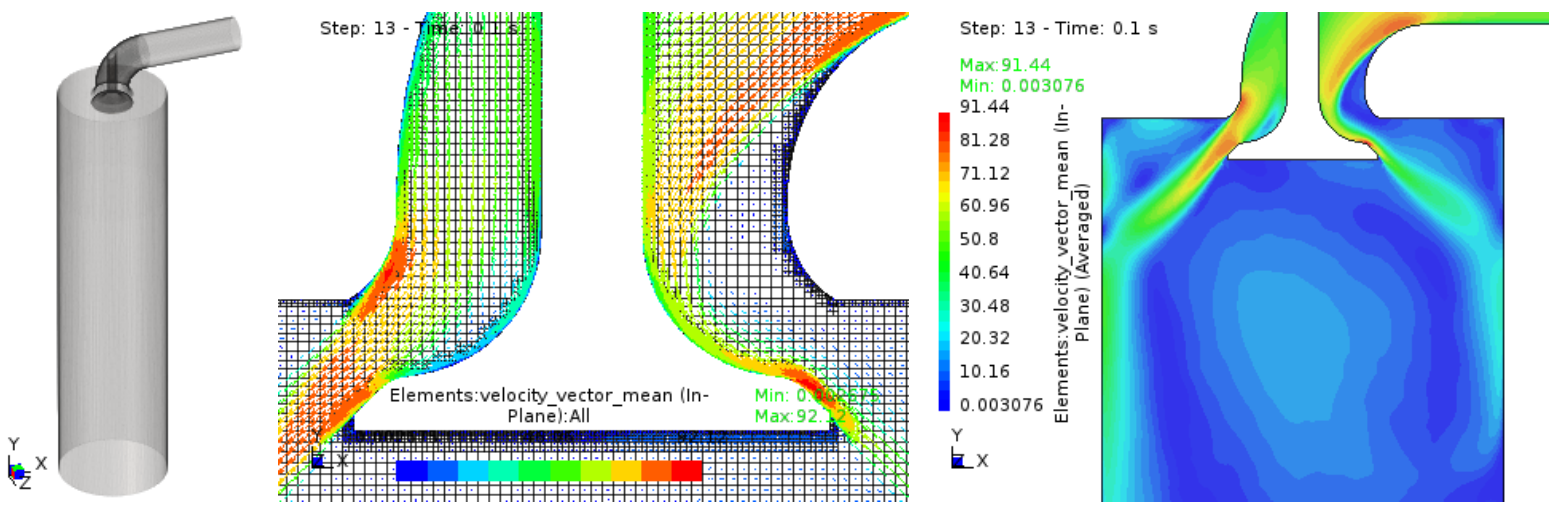

Figure 11: JSAE port. Geometry (left), details of Cartesian cut-cell grid overlaid with timeaveraged velocity vectors (middle) and in-plane time-averaged velocity vector contours (right) around the valve in the symmetry plane $z=0$ (right).

of $p_{\text {out }}=101176 \mathrm{~Pa}$ was specified at the outlet. The measured pressure drop, calculated as the difference between the total pressure at the inlet $p_{t o t}$ and static pressure at the outlet was $\Delta p_{\text {exp }}=3349 P a$.

The Cartesian cut-cell grid with 7.9 million cells and the maximum non-dimensional wall distance of $y_{\max }^{*}=51$ was considered suitable for the present LES. Unsteady runs were performed using the second order TTL time scheme with the time step size of $\Delta t=1 . \times 10^{-5} \mathrm{~s}$. Typically, three outer iterations per time step were required to reduce the normalised residual below the specified convergence criterion of $1 . \times 10^{-5}$. Simulations were conducted over 10,000 time steps $(0.1 \mathrm{~s})$, and the last 5, 000 steps were used to obtain the time-averaged variables.

Figures 11 (middle, right) show velocity plots around the valve in the symmetry plane $z=0$. On the right side of the valve and around the valve seat, a large recirculation region is present. The size of this region is influenced by the flow separation point on the port surface, above the valve seat. Thus this region determines the flow structure in the valve gap, which in turn affects the pressure drop across the port.

The sub-grid scale viscosity at the end of the simulation (as calculated by the UCF-method) is displayed in Figure 12 (left). On the right, time histories of the pressure drop $\Delta p(t)=$ 

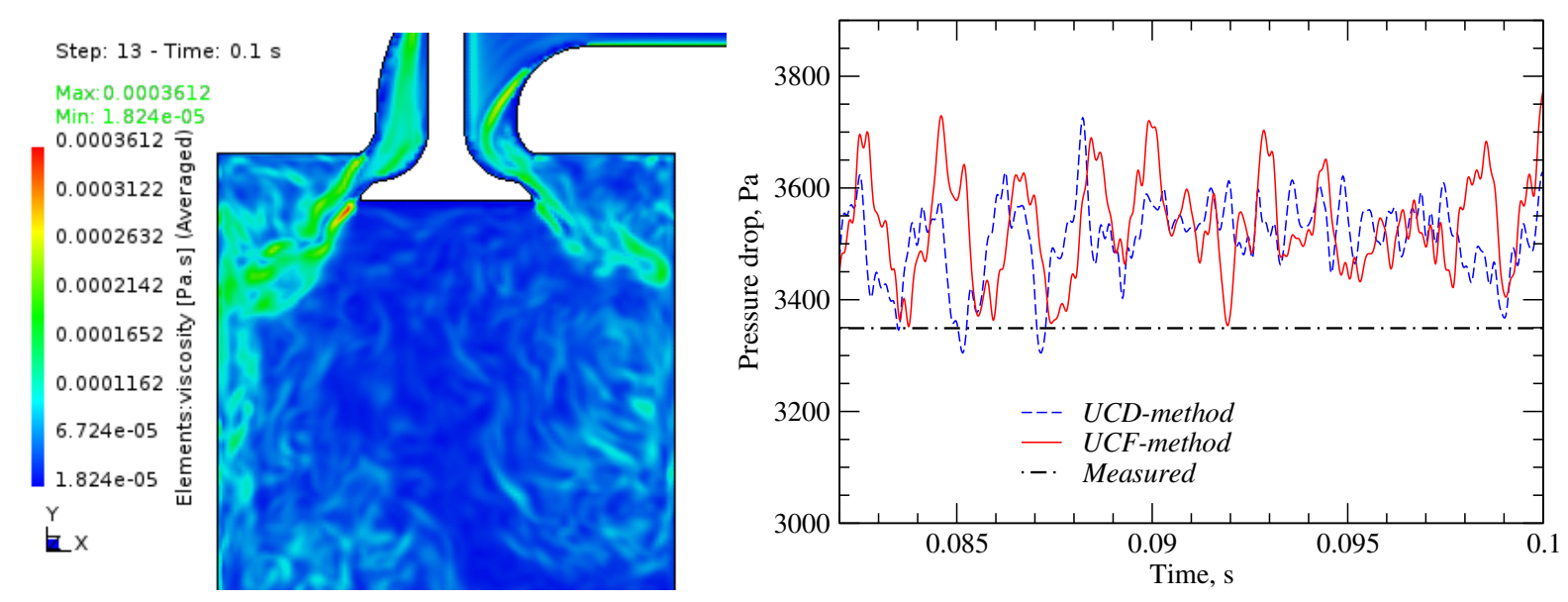

Figure 12: JSAE port. Sub-grid scale viscosity at the given time (left) and the effect of the upwind node reconstruction method on the pressure drop history (right).

$p_{\text {tot }}-p_{\text {out }}$ are plotted for both UCD and UCF upwind node reconstruction methods. The time averaged pressure drop values obtained by UCD and UCF methods are $\Delta p_{C D}=3486 P a$ and $\Delta p_{C F}=3527 P a$, respectively. With reference to the measured pressure drop $\Delta p_{\text {exp }}=$ $3349 \mathrm{~Pa}$, these values over-predict the pressure drop for $4.1 \%$ and $5.3 \%$, respectively.

\section{Conclusions}

The implementation of bounded convective schemes on three-dimensional unstructured grids has been revisited. A central problem of the far upwind node reconstruction is generalised by defining an upwind-biased stencil along the local coordinate connecting the cell centre upstream of the cell-face and the cell-face centre. With this approach, a diffusion-like error introduced by grid skewness can be alleviated. The improved convergence properties and accuracy of the new method are demonstrated by computing four test cases. For each case, either analytical or available experimental/numerical results are used to validate the present method. In addition, the new method is scrutinised against the well established upwind node reconstruction method which by its definition can not mitigate the grid skewness error. Considering the performance of higher order schemes, the MINMOD scheme is well suited for the steady-state simulation whereas the modified SMART is recommended for unsteady simulations. In the case of LES, it is beneficial to employ the bounded central differencing scheme.

\section{References}

[1] Roe, P.L., Characteristic-based schemes for the euler equations. Annual Review of Fluid Mechanics 18:337-365 (1986).

[2] Harten, A., High resolution schemes for hyperbolic conservation laws. Journal of Computational Physics 49:357-393 (1983).

[3] Sweby, P.K., High resolution schemes using flux limiters for hyperbolic conservation laws. SIAM Journal Numer. Anal. 21:995-1011 (1984).

[4] Gaskell, P.H. and Lau, A.K., Curvature compensated convective transport: Smart, a new boundedness preserving transport algorithm. International Journal for Numerical Methods in Fluids 8:617-641 (1988).

[5] Waterson, N.P. and Deconinck, H., Design principles for bounded higher-order convection schemes - a unified approach. Journal of Computational Physics 224:182-207 (2007). 
[6] Darwish, M.S. and Moukalled, F.H., Normalized variable and space formulation methodology for high-resolution schemes. Numerical Heat Transfer B 26:79-96 (1994).

[7] Alves, M.A., Oliveira, P.J. and Pinho, F.T., A convergent and universally bounded interpolation scheme for the treatment of advection. International Journal for Numerical Methods in Fluids 41:47-75 (2003).

[8] Przulj, V.P., Computational Modelling of Vortex Shedding Flows. Ph.D. Thesis, City University, London. http://openaccess.city.ac.uk/id/eprint/7565 (1998).

[9] Piperno, S. and Depeyre, S., Criteria for the design of limiters yielding efficient high resolution tvd schemes. Computers \& Fluids 27:183-197 (1998).

[10] Ubbink, O. and Issa, R.I., A method for capturing sharp fluid interfaces on arbitrary meshes. Journal of Computational Physics 153:26-50 (1999).

[11] Przulj, V. and Basara, B., Bounded convection schemes for unstructured grids. AIAA 2001-2593 (2001).

[12] Jasak, H., Weller, H.G. and Gosman, A.D., High resolution NVD differencing scheme for arbitrarily unstructured meshes. International Journal for Numerical Methods in Fluids 31:431-449 (1999).

[13] Li, L.X., Liao, H.S. and Qi, L.J., An improved r-factor algorithm for TVD schemes. International Journal of Heat and Mass Transfer 51:610-617 (2008).

[14] Hou, J., Simons, F. and Hinkelman, R., A new TVD method for advection simulation on 2d unstructured grids. International Journal for Numerical Methods in Fluids 71:12601281 (2013).

[15] Zhang, D., Jiang, C., Liang, D. and Cheng, L., A review on TVD schemes and a refined flux-limiter for steady-state calculations. Journal of Computational Physics 302:114-154 (2015).

[16] Touze, C.L., Murrone, A. and Guillard, H., Multislope MUSCL method for general unstructured meshes. Journal of Computational Physics 284:389-418 (2015).

[17] Denner, F. and van Wachem, B.G.M., TVD differencing on three-dimensional unstructured meshes with monotonicity-preserving correction of mesh skewness. Journal of Computational Physics 298:466-479 (2015).

[18] Demirdzic, I., Lilek, Z. and Peric, M., A collocated finite volume method for predicting flows at all speeds. Int. Journal for Numerical Methods in Fluids 16:1029-1050 (1993).

[19] Przulj, V. and Basara, B., A SIMPLE-based control volume method for compressible flows on arbitrary grids. AIAA $2002-3289$ (2002).

[20] Rhie, C.M. and Chow, W.L., Numerical study of the turbulent flow past an airfoil with trailing edge separation. AIAA Journal 21:1525-1532 (1983).

[21] Ferziger, J. and Peric, M., Computational Methods for Fluid Dynamics. Springer, Berlin (1997).

[22] Warming, R.F. and Beam, R.M., Upwind second order difference schemes and applications in aerodynamic flows. AIAA Journal 14:1241-1249 (1976).

[23] Leonard, B.P., A stable and accurate convective modelling procedure based on quadratic upstream interpolation. Comp. Meth. Appl. Mech. Eng. 19:59-98 (1979).

[24] van Leer, B., Upwind difference methods for aerodynamic problems governed by the Euler equations. Lectures in Applied Mathematics 22:327-336 (1985).

[25] Demirdzic, I. and Muzaferija, S., Numerical method for coupled fluid flow, heat transfer 
and stress analysis using unstructured moving meshes with cells of arbitrary topology. Comp. Methods Appl. Mech. Eng. 125:235-255 (1995).

[26] Muzaferija, S., Adaptive Finite Volume Method for Flow Predictions Using Unstructured Meshes and Multigrid Approach. PhD dissertation, University of London (1994).

[27] Przulj, V., Birkby, P. and Mason, P., Finite volume method for conjugate heat transfer in complex geometries using Cartesian cut-cell grids. In CHT-08, Marrakech, Morocco (2008).

[28] Patankar, S.V., Numerical Heat Transfer and Fluid Flow. McGraw-Hill, New York (1980).

[29] Van Der Vorst, H.A., Bi-CGSTAB: A fast and smoothly converging variant of Bi-CG for the solution of nonsymmetric linear systems. SIAM J. Scientific Computing 13:631-644 (1992).

[30] Sleijpen, G. and Fokkema, D.R., Bicgstab $(l)$ for linear equations involving unsummetric matrices with complex spectrum. Electronic Trans. Numer. Anal. 1:11-32 (1993).

[31] Przulj, V.P., Generalized SIMPLE-based pressure correction method for unstructured colocated grids. AIAA Journal 0:1-12. Published online March 03, 2016, http: / / dx. doi.org/10.2514/1.J054505 (2016).

[32] Mathur, S.R. and Murthy, J.Y., A pressure based method for unstructured meshes. Numerical Heat Transfer B 31:195-215 (1997).

[33] Leonard, B.P., Simple high-accuracy resolution program for convective modelling of discontinuities. International Journal for Numerical Methods in Fluids 8:1291-1318 (1988).

[34] Georgiadis, N.J., Rizzetta, D.P. and Fureby, C., Large-Eddy Simulation: Current capabilities, recommended practices, and future research. AIAA Journal 48(8):1772-1784 (2010).

[35] Jasak, H., Error Analysis and Estimation in the Finite Volume Method with Applications to Fluid Flows. Ph.D. Thesis, Imperial College, University of London,. (1996).

[36] Demirdzic, I. and Muzaferija, S., Numerical method for coupled fluid flow, heat transfer and stress analysis using unstructured moving meshes with cells of arbitrary topology. Comp. Methods Appl. Mech. Eng. 125:235-255 (1995).

[37] Posdziech, O. and Grundmann, R., A systematic approach to the numerical calculation of fundamental quantities of the two-dimensional flow over a circular cylinder. Journal of Fluids and Structures 23:479-499 (2007).

[38] Williamson, C.H., Oblique and parallel modes of vortex shedding in the wake of a circular cylinder at low reynolds numbers. Journal of Fluid Mechanics 206. 579. (1989).

[39] Przulj, V., Penning, R. and Tiney, N., Conjugate heat transfer modelling of internal combustion engine structures and coolant flows. In CHT-12, Bath, England (2012).

[40] Przulj, V., Pragmatic wall treatment for RANS simulations on Cartesian cut-cell grids. In K. Hanjalic and Y. Nagano (eds.), 6th Int. Symp. Turbulence, Heat and Mass Transfer, Rome, Italy (2009).

[41] Yamada, T., Comparisons of validation of engine intake port flow simulated by commercial CFD codes. JSAE Paper (20014699):20-26 (2001). 